\title{
Foreword by Andreas Graf Gatterburg
}

The AutomationML Initiative was founded in 2006, initiated by Daimler AG. It was intended to generate a neutral data format for engineering data exchange, but a group of pioneers from different companies and universities foresaw the opportunities of data digitalization in the manufacturing industry and developed a data format that is a full-fledged modelling language. Today, 14 years later, AutomationML is a wellknown and accepted technology and already established in industry.

The basic motive of AutomationML is cost savings. Cost-saving is always on the mind of the customer of industrial automation. In our highly developed economies, the cost of skilled labour is most of the time far more significant than material or equipment cost. Nevertheless, all too often engineers are confronted with a situation where they have no possibility to reuse the results of their fellows. A lack of open interconnection between engineers in an automation project leads to exploding costs. Caused by a fragmented documentation and tool landscape, this complex is the cause of many a failed project and hampers the growth of future markets in automation significantly. The story we tell in this book is a different one. It is the story of how openness can make your work better.

When the first book on AutomationML was published a decade ago, AutomationML was considered, even by its inventors, to be nothing but a file format for data exchange in engineering tool chains. It is this still, and an increasingly successful one at that. But, over the years, AutomationML has become more. It has become an information modelling language for automation engineering. AutomationML has, through the tireless work of a committed community, become a host to one of the most extensive pools of engineering models in the industry today.

For industrial companies, the usage of AutomationML has proven to be doubly beneficial. Not only can the application of AutomationML help diminish data loss and resulting incompatibilities in engineering tool chains, but it was also proven that modelling information in AutomationML is a future-proof approach to support the digitalization of plants, products and portfolios.

If, as a reader, you now wonder if the claims laid down in this preface can possibly be true, I cordially invite you to make your own assessment. In this book, you find the current state of the art for AutomationML to the best of the knowledge of global experts for the technology. The AutomationML e.V. as the caretaker for the AutomationML technology would also like to invite you to engage with us and give us feedback on your thoughts about what you will read in this book. And, maybe, to join us in the future journey of the AutomationML technology.

Dr.-Ing. Andreas Graf Gatterburg, Hattersheim, November 2020 First Chairman of the Board of AutomationML e.V. 
